\title{
DeliData: A dataset for deliberation in multi-party problem solving
}

Georgi Karadzhov, Tom Stafford, Andreas Vlachos

\section{Task - Wason Card Selection}

- Extensively studied in psychology for group decision making

- Most people get it wrong at first $(20 \%$ success rate)

- By engaging in conversation groups solve it better - $70-80 \%$ success rate
2. Experimental Setup:

- We developed a platform for collecting group dialogues in-the-wild

- Each experiment run contains 3 phases: (i) participants venture a solo solution, (ii) then engage in a group discussion, finally (iii) submit a revised solution

\section{\begin{tabular}{ll|l|l|}
$U$ & 2 & $Z$ & 3
\end{tabular}}

Each of the 4 cards below has a letter on one side and a number on the other. Which card(s) do All cards with vowels on one side have an even number on the other.

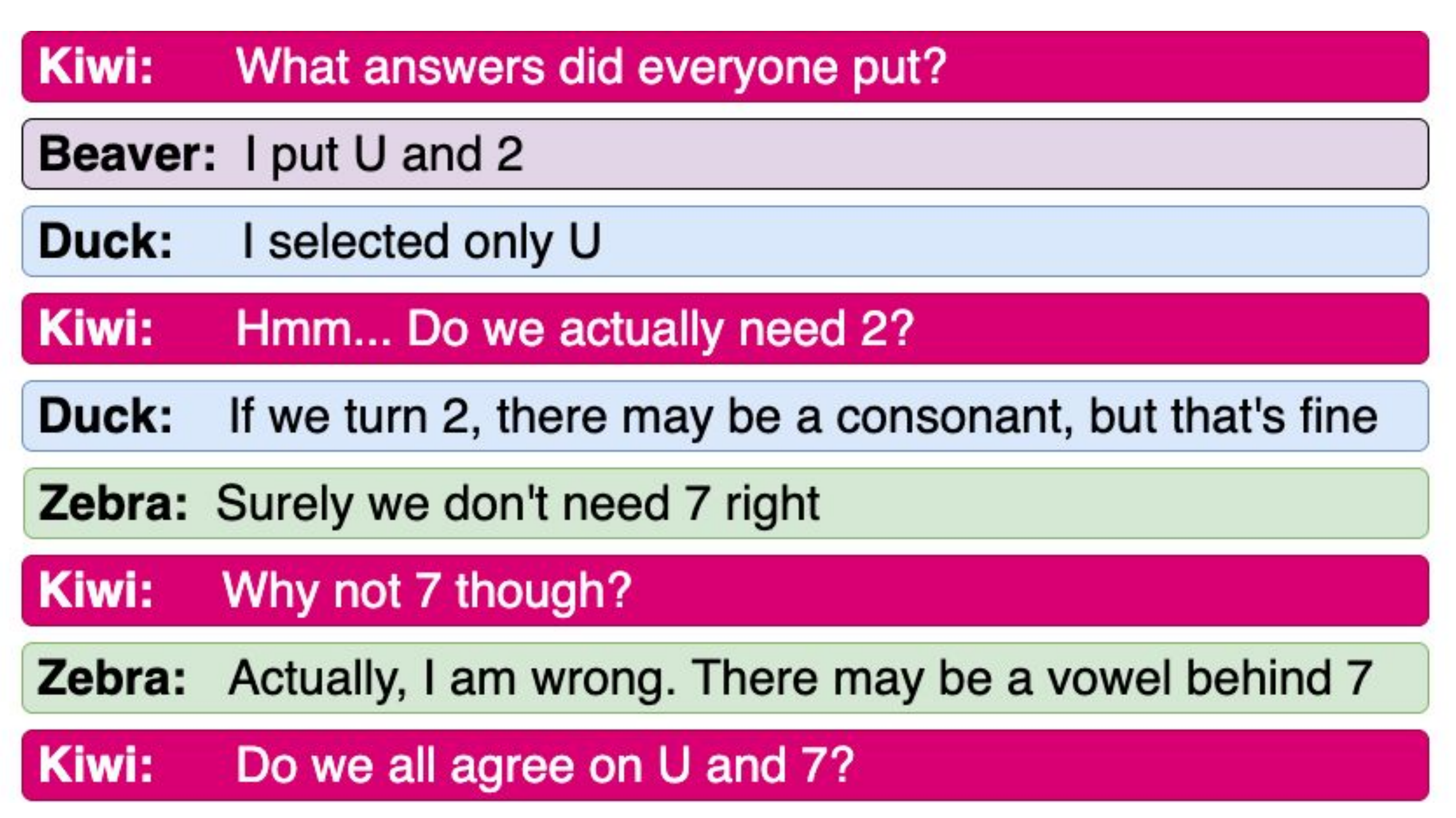

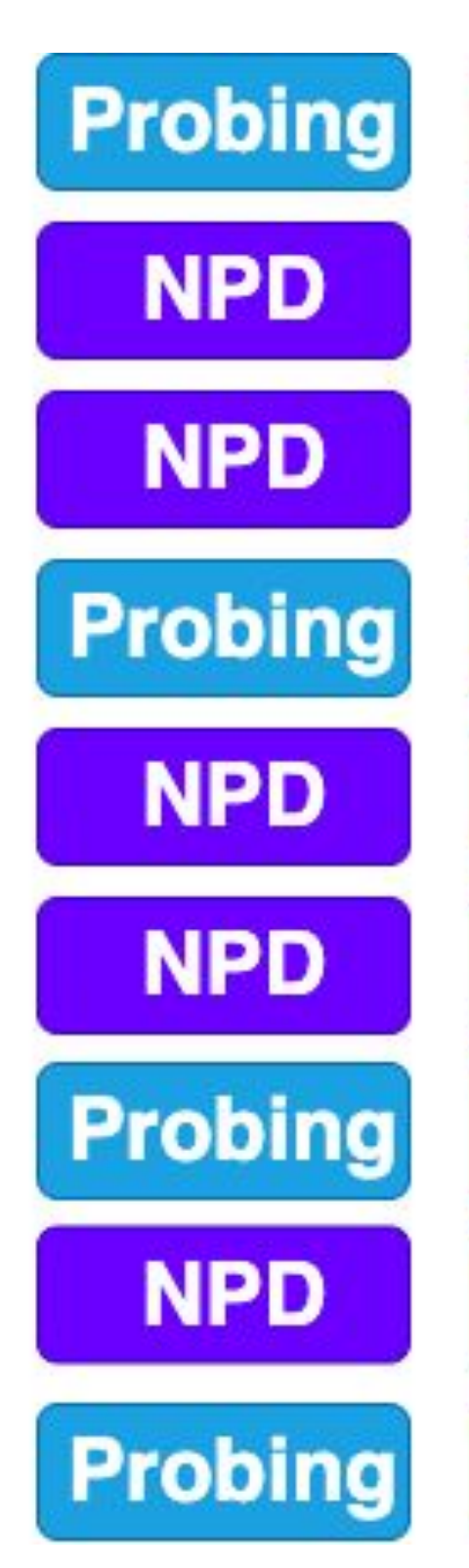

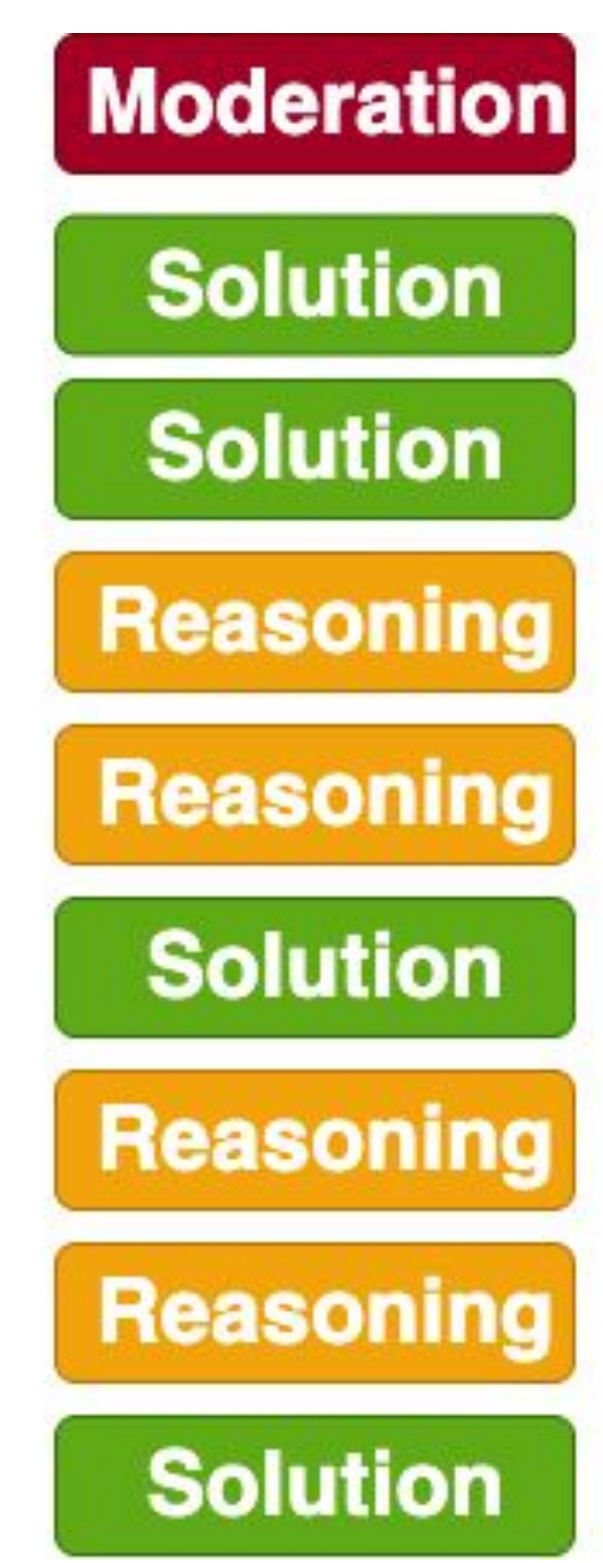

complete_solution complete_solution

partial_solution consider_opposite

solution_summary
3. In-lab and in-the-wild data collection: DeliData will be the first publicly available dataset on group collaboration

\begin{tabular}{|l|l|l|l|}
\hline & In-lab & In-the-wild & Total \\
\hline Number of Dialogues & 17 & 209 & 226 \\
\hline Total Participants & 50 & 658 & 708 \\
\hline AVG utterances & 41.05 & 28.7 & 29.6 \\
\hline AVG utterance length & 8.3 & 7.95 & 7.98 \\
\hline Solo-performance & 0.61 & 0.59 & 0.59 \\
\hline Group Performance & 0.84 & 0.70 & 0.71 \\
\hline Agreement & 0.91 & 0.82 & 0.83 \\
\hline
\end{tabular}

Thanks to the Isaac Newton Trust and Cambridge University Press, we will be able to collect 360 more dialogues.

\section{The Future: Deliberation} Enhancing Bots (DEliBots)

- Dialogue systems that participate in the group discussion in order to improve the group performance through deliberation

- The DEliBot doesn't know the answer to the task, but knows how to ask good probing questions length and number of participants
Success, compared to dialogue

\begin{tabular}{|l|l|}
\hline Feature & Correlation \\
\hline Probing-Reasoning & 0.119 \\
\hline \# of participants & 0.056 \\
\hline Dialogue Length & 0.077 \\
\hline
\end{tabular}

- Participants actively engage in statements

Inter-annotator agreement: 0.75 annotation, and 0.53 for the additional labels

- Stronger correlation between Probing for reasoning and

\section{Let's talk about constructive discussions:}

gmk34@cam.ac.uk

\begin{tabular}{|l|l|l|l|l|l|l|}
\hline Moderation & 89 & 0 & 89 & specific_addressee & 55 & 4.4 \\
\hline Reasoning & 59 & 453 & 512 & complete_solution & 258 & 20.7 \\
\hline Solution & 66 & 305 & 371 & partial_solution & 79 & 6.3 \\
\hline Agree & 0 & 265 & 265 & solution_summary & 40 & 3.2 \\
\hline Disagree & 0 & 9 & 9 & consider_opposite & 11 & 0.9 \\
\hline Total & 214 & 1032 & 1246 & connnnnnn
\end{tabular}

